\title{
Characterization and Stability of Silver Nanoparticles in Aqueous Solutions
}

\author{
L. H. Bac ${ }^{a, b}$, W. H. Gu ${ }^{a}$, J. C. Kim ${ }^{a, *}$, B. K. Kim ${ }^{a}$, and J. S. Kim ${ }^{a}$ \\ ${ }^{a}$ School of Materials Science Engineering, University of Ulsan, Daehak-ro 102, \\ Nam-gu, Ulsan, 680-749Korea \\ ${ }^{b}$ School of Engineering Physics, Hanoi University of Science and Technology, \\ No. 1 Dai Co Viet Road, Hanoi, Vietnam \\ (Received January 13, 2012; Revised January 30, 2012; Accepted February 8, 2012)
}

\begin{abstract}
In this work, the silver nanoparticles have been synthesized by electrical explosion of wire in three liquid mediums: deionized water (DIW), polyvinylpyrrolidone (PVP) and sodium dodecyl benzene sulfonate (SDBS) solutions. Absorption in the UV-visible region of these suspensions was measured in the range of 300-800 nm. A surface plasmon peak was observed at $\sim 400 \mathrm{~nm}$ in all suspensions in measured wavelength range. Particle size was analyzed by transmission electron microscope. It showed that the particles had nearly spherical shape in all samples. The average particle sizes prepared in DIW, PVP and SDBS solution were 37, 31 and $27 \mathrm{~nm}$, respectively. Stability of the suspensions was estimated by multiple light scattering method. The presence of PVP and SDBS surfactants in the exploding medium resulted in enhanced stability of the silver suspensions.
\end{abstract}

Keywords: Silver nanoparticles, Electrical explosion of wire (EEW), multiple light scattering, Stability

\section{Introduction}

Noble metal nanocolloids have been attracted much attention in recent years due to their specific chemical and physical behaviors. They have widely investigated for applications in many fields: electronics, catalysis, sensing, drug delivery system and biological labeling [1-5]. Many methods are available to synthesize the noble metal nanocolloids including physical and chemical approaches such as chemical reduction [6], electrochemistry [7], laser ablation [8], gamma irradiation [9], and electrical arc discharge [10]. Among physical methods, electrical explosion of wire (EEW) in liquid is an attractive method because of simplicity, low impurity introduction, and low cost [11]. Vacuum apparatus is no need in this process. Moreover, it is easy to scale up for mass production. The products of wire explosion process depend on the current density, the wire dimension and the medium in which explosion is carried out. The ambient environment is one of the important parameters of this method. Non-noble metals exploded can react with the molecules of liquid to form a new phase $[12,13]$. Noble metals do not react with the ambient medium but surface modification of nanoparticles and quality of products are much dependent on the environment. Ambient liquid properties affect on the explosion and particles formation and therefore they can be used to control the properties of colloids. The stability of gold colloids obtained by EEW in water increased at higher liquid temperature although mean size of particles became bigger [14]. In contrast, silver colloids at lower synthetic temperature have better dispersion properties than those at higher temperatures [15]. The size of nanoparticles and their properties significantly changed when surfactant presented in the working liquid [14]. Evidently, surfactant plays an important role in stability of suspension and properties of the nanoparticles. Particle size is smaller and size distribution is narrower when exploding in surfactant medium. Stability of colloids is much better with higher zeta potential value and lower sedimentation rate [16].

*Corresponding Author: J. C. Kim, TEL: +82-52-259-2231, FAX: +82-52-259-1688, E-mail: jckimpml@ulsan.ac.kr 
The present paper reported the characterization and stability of silver colloids obtained in various aqueous environments. This result is a series of our work on the noble metal colloids which fabricated by EEW in liquid. Effects of water and two kinds of surfactant (PVP and SDBS) on the particle size, optical property and stability of silver colloids were investigated. We observed that the presence of surfactant in the working liquid could dramatically enhance stability of the silver colloids.

\section{Experiment Details}

Silver colloid was prepared by EEW in liquid from wire with $2 \mathrm{~mm}$ in diameter. Experimental setup has been described elsewhere [17]. PVP and SDBS surfactants were dissolved in the deionized water with concentration of $0.01 \mathrm{M}$. These solutions were used as working liquid for explosion process. The $400 \mathrm{ml}$ liquid was filled in a teflon beaker with $73 \mathrm{~mm}$ in diameter and $124 \mathrm{~mm}$ in height. A section of silver wire $(25 \mathrm{~mm})$ was installed between two electrodes and submersed in liquid. A pulsed high-density current from a $3 \mathrm{kV}$ charged capacitor was injected through the wire by closing the spark gap switch. Because the resistance of liquid is much smaller than that of silver, the electrical energy only deposited on the wire. Silver wire is rapidly heated to form vapor and plasma due to a high rate of the energy injection and an expansion lag of the heated material. Consequently, a bright light flashes and a shockwave scatters to the ambient atmosphere. The silver vapor was rapidly cooled down when colliding with the liquid molecules and condensed into solid particles dispersed directly in the liquid. The experimental parameters of explosion process were shown in Table 1. After 25 times of explosion, the suspension was collected for analysis.

The morphology and the size of the prepared silver nanoparticles were observed by transmission electron microscopy (TEM). A drop of silver suspension was dropped on a carbon coated copper grid then dried under vacuum. The absorption spectrum of silver colloids was investi-

Table 1. Experimental details of EEW in liquid

\begin{tabular}{ccccc}
\hline $\begin{array}{c}\text { Capacitance } \\
(\mu \mathrm{F})\end{array}$ & $\begin{array}{c}\text { Charging } \\
\text { voltage } \\
(\mathrm{kV})\end{array}$ & $\begin{array}{c}\text { Wire } \\
\text { diameter } \\
(\mathrm{mm})\end{array}$ & $\begin{array}{c}\text { Wire } \\
\text { length } \\
(\mathrm{mm})\end{array}$ & $\begin{array}{c}\text { Aqueous } \\
\text { medium }\end{array}$ \\
\hline 30 & 3 & 0.2 & 25 & $\begin{array}{c}\text { Water, PVP, } \\
\text { SDBS }\end{array}$ \\
\hline
\end{tabular}

gated by UV-vis method in the wavelength range of 300$800 \mathrm{~nm}$. The dispersion stability of silver colloids was estimated using a Turbiscan Lab (Formulaction Co., France) based on multiple light scattering method. The apparatus has an optical detection head composed of a pulsed nearinfrared light source and two synchronous detectors. The transmission detector receives the light which passes through the sample while backscattering detector receives the light scattered backward by the sample. The dispersion was contained in a $30 \mathrm{ml}$ cylindrical glass measurement cell. The detection head scans the entire height of the sample, recording the transmission and backscattering signal as a function of the sample height in a step of $40 \mathrm{~mm}$ every $2 \mathrm{~h}$ for 3 days. Zeta potential property of silver nanocolloids was measured by zeta potential analyser (ELS-Z, Japan). At least ten different runs were carried out to take the average zeta potential value.

\section{Results and Discussion}

Fig. 1 illustrates the TEM images and corresponding size distributions of silver nanoparticles produced by EEW in DIW, PVP and SDBS solutions. The results show that the particle shape in all cases is nearly spherical. These results are in agreement with one absorption peak in optical spectrum (Fig. 2). The average particles sizes were calculated from TEM images to be 28,31 and $37 \mathrm{~nm}$ in the SDBS, PVP and DIW, respectively. Particle size in DIW is biggest and particles are more aggregated. The average diameter of nanoparticles decreases when nanoparticles were prepared in surfactant solutions. Moreover, the size distribution also become narrower with presence of surfactant. In SDBS solution, the particle size is smallest and size distribution is narrowest in three samples.

Fig. 2 shows absorption spectra of silver colloid prepared by EEW in different aqueous media. All spectra exhibit a peak at around $400 \mathrm{~nm}$, which is related to the surface plasmon resonance of silver nanoparticles and a tail of a broad band extending toward the UV wavelength. It is noted that number of peaks in absorption spectrum indicates the shape of particles; one plasmon absorption band is characteristic of a spherical nanoparticle and two plasmon absorption bands is characteristic of an ellipsoidal/nanorod particle [18]. The single surface plasmon peak of these spectra implies that the silver nanoparticles fabricated by EEW are spherical. The detail characteristics of silver absorption spectra in DIW, PVP and SDBS were listed in 

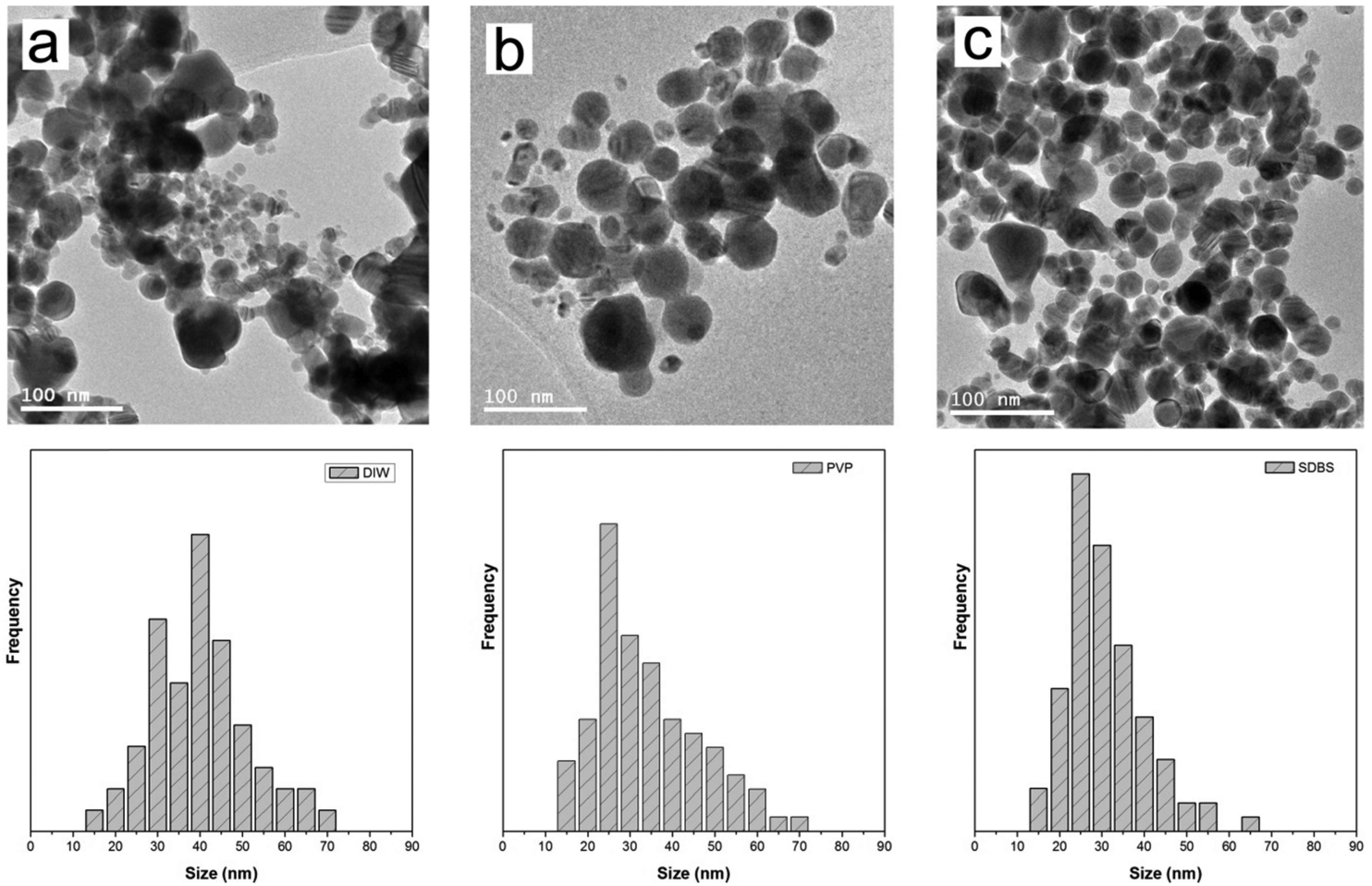

Fig. 1. TEM images and corresponding size distributions of nanoparticles prepared in different media: (a) DIW, (b) PVP and (c) SDBS.

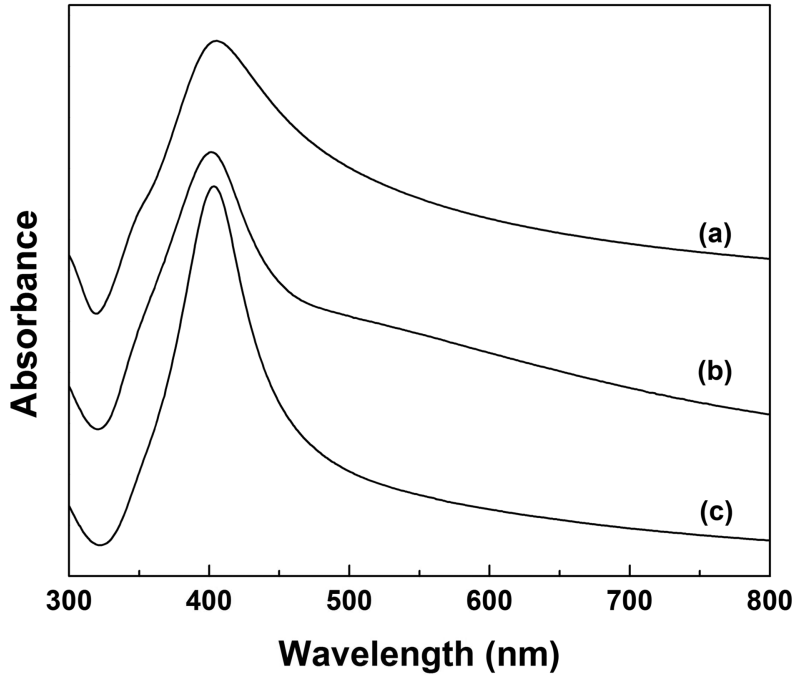

Fig. 2. UV-vis absorption spectra of silver colloidal solution prepared at different media: (a) DIW, (b) PVP, and (c) SDBS.

the Table 2. Position of peak shifts a small value with changing the liquid but the width of surface plasmon peak dramatically varies. According to the Mie theory, the peak shift and the change of peak width are observed when the particle size and size distribution change [19]. The absorption peaks of silver nanoparticles in SDBS and PVP are similar while it is red shift in the DIW. The peak width of silver nanoparticles in PVP is broader than
Table 2. Characteristics of silver nanoparticles prepared by EEW in various aqueous solutions

\begin{tabular}{lcrcrl}
\hline \hline Sample & $\begin{array}{c}\text { Peak position } \\
(\mathrm{nm})\end{array}$ & FWHM & $\begin{array}{c}\text { Particle Z-potential } \\
\text { size }(\mathrm{nm})\end{array}$ & $\begin{array}{l}\text { (mV) } \\
\text { Dispersion state }\end{array}$ & \\
\hline SDBS & 403 & 71 & 28 & -34.83 & Stable colloid \\
PVP & 402 & 82 & 31 & -29.16 & Stable colloid \\
DIW & 406 & 108 & 37 & -15.21 & Unstable colloid \\
\hline
\end{tabular}

that of silver particles in SDBS. The broader peak in PVP than in SDBS indicates that the silver particles have larger range size. Although the peak position of silver particles in PVP is little smaller, the average size is still bigger due to larger size distribution. In case of DIW, the significantly broader bandwidth and red-shift of absorption peak surely confirm the biggest particles size and the widest size distribution.

To investigate the stability of silver nanocolloids, we measured their zeta potential and multi light scattering. The high value of zeta potential makes a repulsion force and keeps the nanoparticles away from each other, which results in a high stability of colloid. Table 2 shows the zeta potential of silver nanocolloids prepared by EEW in different aqueous media. The all silver nanoparticles are negatively charged with zeta potential value in the range of $-15 \mathrm{mV}$ to $-35 \mathrm{mV}$. It is found that the absolute zeta 
potential value increases significantly when surfactants were used in the exploding media. The colloid prepared in SDBS solution has higher zeta potential value. The zeta potential of the particle surface in DIW is at the minimum among three samples. The dispersion stability of this colloid is poor because the force of electrostatic repulsion between particles is not sufficient to overcome the attraction force between particles. The zeta potential of the surface particles increases when surfactants are in liquid. Consequently, the electrostatic repulsion force between particles is sufficient to prevent attraction and collision between particles. The higher electrostatic forces resulted in the steric forces that generate by the absorption of surfactant molecules on the surface of particles. Therefore, colliding among particles and agglomeration of particles can be more difficult and the dispersion stability of silver nanoparticles in surfactant is the better than that of particles in water.

Fig. 3 presents transmission (T) profiles as a function of sample height and time measured in three days. The transmitted light flux in percentage (\%) related to the density of suspension in the liquid. The change of nanoparticle concentration affects on the transmitted light flux. The less particles cause the more transmission light going across the sample. The initial transmission scan was used for reference and set to be $0 \%$ transmission signal throughout the length of the measured cell.

It can be seen clearly that the transmission profile of silver suspension in water shows clarification of sample with increasing $\mathrm{T}$ level on the right of profile. This phenomenon occurs due to the particle migration. Silver particles migrating from the top to the bottom induce particle volume fraction change on the extremities of sample. As characterizing in zeta potential measurement,

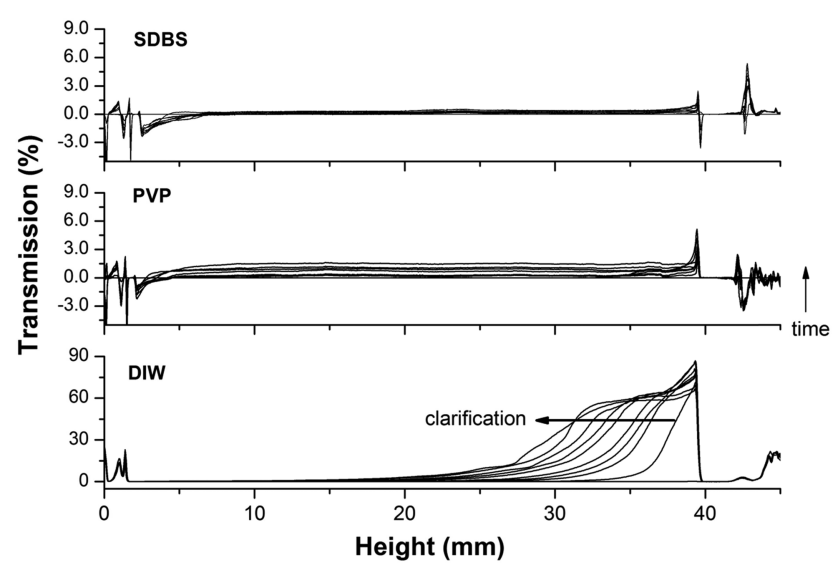

Fig. 3. Transmission profile of silver colloids prepared in different exploding media. the nanoparticles in water have small surface charge. As a result, the electrostatic forces between particles are low and repulsive forces between particles are not high enough to prevent the collision and attractive forces among particles. Consequently, the particles coagulation can be happened and then settled due to big aggregated cluster. The particles sediment reduces the volume fraction and causes the increasing transmission signal. The transmission profile of suspensions with SDBS and PVP surfactant has similar behavior and is very different from $\mathrm{T}$ profile of suspension in DIW. The transmission signal does not change over total height of the sample in each scan. This means that the volume fraction is the same in all area of sample at the scanning time. The transmission increases slightly with time because the suspension settling takes place. However, the settling speed is very slow and no clarification can be found in the each scan after 3 days.

Fig. 4 compared the variation of the $\mathrm{T}$ signal as a function of time for silver suspensions prepared in DIW, SDBS and PVP. From this figure, it indicates that the variation of $\mathrm{T}$ signal is much depends on working liquid of EEW. In other words, the stability of silver suspension is influence by the working liquid. In all cases, $\mathrm{T}$ variation increases as a function of time but the speed of variation is different. It shows clearly that the silver colloid in surfactant solution is much more stable than that in DIW due to the much smaller variation of transmission signal. The $\mathrm{T}$ variation of suspension in water can be divided into three zones. The first zone less than $10 \mathrm{~h}$, the particles migrated slowly corresponding small slope of $\mathrm{T}$ variation. In zone II, the variation of $\mathrm{T}$ signal

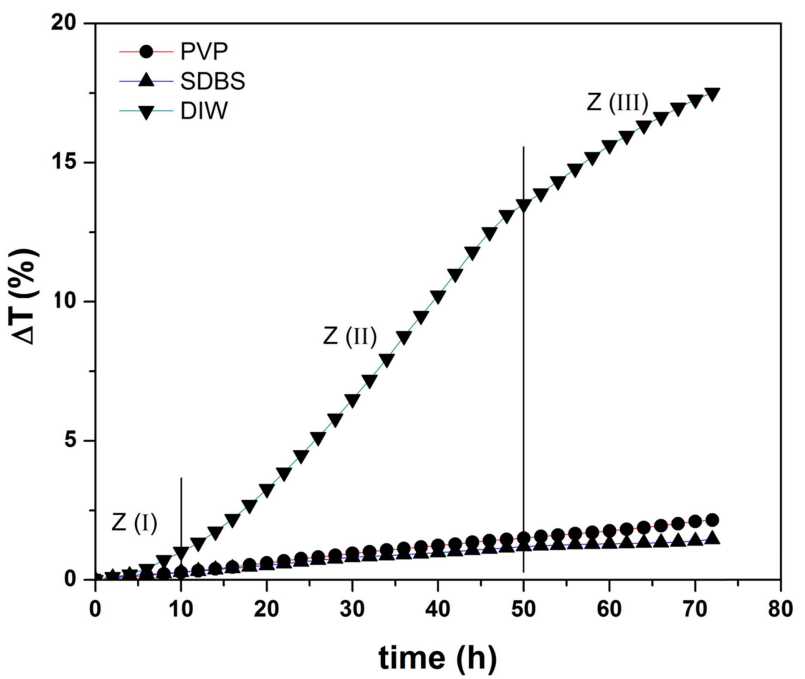

Fig. 4. Variation of transmission signal (DT) vs. time for silver colloids produced by EEW in different aqueous media. 


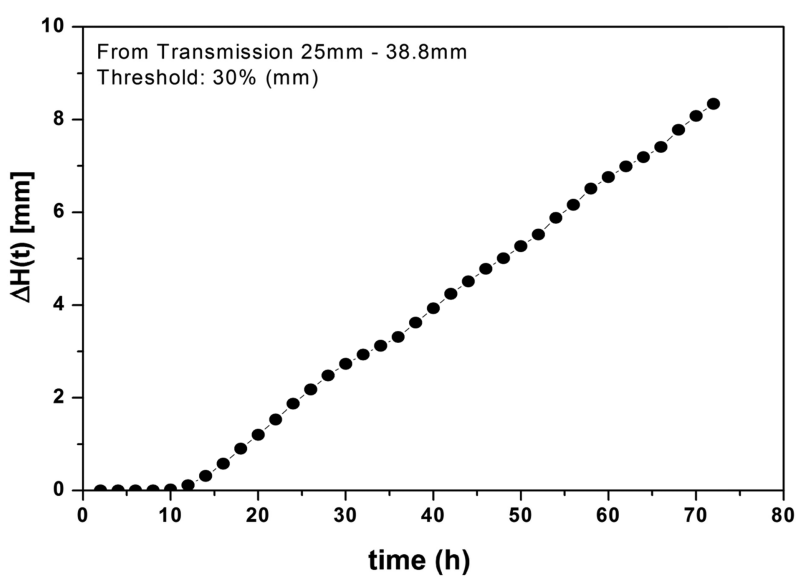

Fig. 5. Thickness of clarification layer of silver colloid prepared in DIW.

increases faster with higher slope of curve. Slope of clarification kinetics are significant due to the high velocity of particle movement and this means that the sedimentation rate is rapid in this period. In zone III, the slope and the sedimentation rate decreases. It is easy to observe that the transmission flux increases at the top of the sample from the T profile of silver particles in DIW. The thickness (delta $\mathrm{H}$ ) of the clarification layer taken out from the transmission in the $25-38 \mathrm{~mm}$ with threshold of $30 \%$ transmission flux is shown in Fig. 5. It was small change less than $10 \mathrm{~h}$ and became considerably afterward. It changed linearly following the time and was about $8 \mathrm{~mm}$ after 72 h. The variation of silver suspension in surfactant is very different in DIW. No clarification is observed in transmission scan. The transmission flux slightly increased with time through the total height of the sample. The change of $\mathrm{T}$ level of silver colloids with presence of surfactant is much smaller than that in DIW. It is about $1.5 \%$ and $2.2 \%$ corresponding to particles in SDBS and PVP, while it is $17.5 \%$ in DIW. The small change of transmission flux deduces that the stability of suspension in surfactant is much better than that in water.

\section{Conclusion}

Electrical explosion of wire in liquid was employed to prepare silver colloids in DIW, PVP and SDBS solutions. The working liquid of EEW much affects on the properties of the colloids. Particles in DIW, PVP and SDBS all are in nearly spherical shape. The nanoparticle size is smaller and size distribution is narrower when SDBS and PVP surfactant were used. Optical analysis in the range of 300-800 nm exhibited an absorption peak at $\sim 400 \mathrm{~nm}$. The peak position of silver nanoparticles in water was red-shifted and the peak width in water was broader than that in surfactant. The stability of suspension was investigated by multi light scattering. The results showed that the stability of silver particles strongly depends on the exploding medium. Dispersion property is instable in the DIW and is much better in PVP and SDBS solutions. The best stability among three samples is SDBS sample with the highest zeta potential value and the lowest speed of transmission variation.

\section{References}

[1] Y. N. Li, Y. L. Wu and B. S. Ong: J. Am. Chem. Soc., 127 (2005) 3266.

[2] Y. C. Kim, N. C. Park, J. S. Shin, S. R. Lee, Y. J. Lee and D. J. Moon: Catal. Today, 87 (2003) 153.

[3] D. V. Talapin, J. S. Lee, M. V. Kovalenko and E. V. Shevchenko: Chem. Rev., 110 (2010) 389.

[4] D. P. O'Neal, L. R. Hirsch, N. J. Halas, J. D. Payne and J. L. West: Cancer Lett., 209 (2004) 171.

[5] D. M. K. Lam and B. W. Rossiter: Sci. Am., 245 (1991) 48.

[6] P. V. Kamat: J. Phys. Chem. B, 106 (2002) 7729.

[7] J. Zhu, S. Liu, O. Palchik, Y. Koltypin and A. Gedanken: Langmuir, 16 (2000) 6396.

[8] F. Mafune, J. Y. Kohno, Y. Takeda, T. Kondow and H. Sawabe: J. Phys. Chem. B, 104 (2000) 8333.

[9] W. T. Wu, Y. Wang, L. Shi, Q. Zhu, W. Pang, G. Xu and F. Lu: Nanotechnology, 16 (2005) 3017.

[10] J. K. Lung, J. C. Huang, D. C. Tien, C. Y. Liao, K. H. Tseng, T. T. Tsung, W. S. Kao, T. H. Tsai, C. S. Jwo, H. M. Lin and L. Stobinski: J. Alloys Compd., 434/435 (2007) 655.

[11] Y. A. Kotov: J. Nanopart. Res., 5 (2003) 539.

[12] A. P. Ilyin and O. B. Nazarenko: J. Optoelectron Adv. M., 9 (2007) 1521.

[13] N. Goswami and P. Sen: J. Nanopart. Res., 9 (2007) 513.

[14] L. H. Bac, G. S. Yun, J. S. Kim, H. S. Choi and J. C. Kim: J. Nanosci. Nanotechno., 11 (2011) 1730.

[15] G S. Yun, L. H. Bac, J. S. Kim, Y. S. Kwon, H. S. Choi, J. C. Kim: J. Alloys Compd., 509 (2011) S348.

[16] L. H. Bac, K. S. Kim, J. S. Kim, J. C. Kim and C. K. Rhee: J. Kor. Powd. Met. Inst., 17 (2010) 464 (Korean).

[17] L. H. Bac, J. S. Kim and J. C. Kim: Res. Chem. Intermed., 36 (2010) 795.

[18] S. Link and M. A. E. Sayed: J. Phys. Chem. B, 103 (1999) 8410.

[19] U. Kreibig and M. Vollmer: Optical properties of metal clusters, Springer, Berlin, (1995).

이 논문을 지난 30 여 년간 한국분말야금학회 발전 및 기술개발에 큰 업적을 남기신 울산대학교 권영순 교수님의 정년을 기념하여 헌정합니다. 